Classification

Physics Abstracts

$05.40-68.70$

\title{
Some observations on kinetic walk models
}

\author{
L. Peliti \\ Dipartimento di Fisica, Università « La Sapienza », Piazzale Aldo Moro 2, I-00185 Roma, Italy
}

(Reçu le 18 juin 1984, accepté le ler août 1984)

\begin{abstract}
Résumé. - Les chemins cinétiques sont des chemins qui se développent dans le temps et interagissent avec leur propre passé. On introduit une formulation en théorie des champs pour certains de ces chemins et on en discute les conséquences pour leur comportement asymptotique.
\end{abstract}

Abstract. - Kinetic walks are walks which develop in time and interact with their own past. A field theoretical formulation is introduced for some of these walks' and its consequences for their asymptotic behaviour are sketched.

\section{Introduction.}

A fair amount of kinetic walk models have recently been introduced. They have the common feature of considering walks developing randomly as time goes on, with statistical weights which, at each step, depend on the past history. One of the first examples was the " true " self-avoiding walk (TSAW), which tries to avoid places it has already visited [1]. More recently a kinetic growth walk (KGW) has been introduced, which has the feature of terminating whenever it arrives at a lattice site (a «trap ») whose neighbours have all been visited [2,3]. This model may work as a description of polymer growth. A further refinement of $\mathrm{KGW}$ has also been suggested, which is able to recognize " traps » before falling into them [4].

Most of the studies of these models made use either of exact enumeration of all walks of small length [2-4], or of computer simulation [1, 2]. The experience of TSAW shows however that renormalization group methods may be useful. It was shown in fact [5] that the renormalization scheme of TSAW is fairly complicated, yielding a crossover in its asymptotic behaviour which is hard to spot on the bases of either perturbation theory or of simulation alone.

It is therefore worthwhile to investigate ways of introducing field theories to represent these models, and to analyse them by standard renormalization group tools. An attempt in this direction for the TSAW [5] yielded indeed as a bonus an acceptable description of the random walk in a random medium $[6,7]$. It is also easy to generalize the models at the level of their field theoretical description, as was shown by the introduction of pseudoscalar interactions in the TSAW [8]. We shall expound in the following the steps leading to a field theory for some forms of kinetic walks. 


\section{Kinetic self-avoiding walk.}

Let us first consider the trivial problem of a random walk in presence of a preassigned density $\phi(\mathbf{r}, t)$ of traps. We shall actually assume that the walk $\mathbf{R}(t)$ terminates at time $t$ with a probability $g \phi(\mathbf{R}(t), t)$, where $g$ is a small parameter. This corresponds to traps with low efficiency. It is then easy to write down a perturbative series in $g$ for the probability $G\left(\mathbf{r}, t ; \mathbf{r}_{0}, t_{0}\right)$ that a walk which originates from $\mathbf{r}_{0}$ at time $t_{0}$ reaches $\mathbf{r}$ at time $t$ :

$$
\begin{aligned}
& G\left(\mathbf{r}, t ; \mathbf{r}_{0}, t_{0}\right)=G_{0}\left(\mathbf{r}, t ; \mathbf{r}_{0}, t_{0}\right) \\
& -g \int_{t_{0}}^{t} \mathrm{~d} t_{1} \int \mathrm{d} \mathbf{r}_{1} G_{0}\left(\mathbf{r}, t ; \mathbf{r}_{1}, t_{1}\right) \phi\left(\mathbf{r}_{1}, t_{1} ; \mathbf{r}_{0}, t_{0}\right) G_{0}\left(\mathbf{r}_{1}, t_{1} ; \mathbf{r}_{0}, t_{0}\right) \\
& +g^{2} \int_{t_{0}}^{t} \mathrm{~d} t_{1} \int_{t_{1}}^{t} \mathrm{~d} t_{2} G_{0}\left(\mathbf{r}, t ; \mathbf{r}_{2}, t_{2}\right) \phi\left(\mathbf{r}_{2}, t_{2}\right) G_{0}\left(\mathbf{r}_{2}, t_{2} ; \mathbf{r}_{1}, t_{1}\right) \phi\left(\mathbf{r}_{1}, t_{1}\right) G_{0}\left(\mathbf{r}_{1}, t_{1} ; \mathbf{r}_{0}, t_{0}\right)+\cdots
\end{aligned}
$$

where $G_{0}\left(\mathbf{r}, t ; \mathbf{r}_{0}, t_{0}\right)$ is the corresponding quantity for an ordinary, trap-free, random walk. Equation (1) corresponds to an integral equation for $G$ of the form of Dyson's equation, whose solutions are the retarded solutions of the differential equation :

$$
\left[\frac{\partial}{\partial t}-\mathfrak{D} \nabla^{2}+g \phi(\mathbf{r}, t)\right] G\left(\mathbf{r}, t ; \mathbf{r}_{0}, t_{0}\right)=\delta\left(t-t_{0}\right) \delta\left(\mathbf{r}-\mathbf{r}_{0}\right),
$$

in which $\mathfrak{D}$ is the diffusion constant of the random walk.

One may obtain $G$, as expressed by equation (1), as the propagator of a field theory of the Martin-Siggia-Rose [9] type, in which a pair of real, canonically conjugate fields $\hat{\psi}, \psi$ appear [6]. The field $\hat{\psi}$ acts as a generator of walks, and $\psi$ as an annihilator. The Hamiltonian of this field theory reads :

$$
\mathscr{H}=\mathscr{H}_{0}+\mathscr{H}_{1}
$$

where

$$
\begin{aligned}
& \mathscr{H}_{0}=\int_{t_{0}}^{\infty} \mathrm{d} t \int \mathrm{d} \mathbf{r} \hat{\psi}(\mathbf{r}, t)\left(-\frac{\partial}{\partial t}+\mathscr{D} \nabla^{2}\right) \psi(\mathbf{r}, t), \\
& \mathscr{H}_{1}=\int_{t_{0}}^{\infty} \mathrm{d} t \int \mathrm{d} \mathbf{r} \hat{\psi}(\mathbf{r}, t) g \phi(\mathbf{r}, t) \psi(\mathbf{r}, t) .
\end{aligned}
$$

Similarly to what has been done for the TSAW, we may now consider $\phi(\mathbf{r}, t)$ as a dynamically developing quantity, related at each time to the past history of the walk. The simplest case is the one in which $\phi(\mathbf{r}, t)$ is simply proportional to the density $\rho(\mathbf{r}, t)$ of points already visited, which is represented by our field theory in the following way :

$$
\rho(\mathbf{r}, t)=\int_{t_{0}}^{t} \mathrm{~d} t_{1} \hat{\psi}\left(\mathbf{r}, t_{1}\right) \psi\left(\mathbf{r}, t_{1}\right) .
$$

If we absorb the proportionality constant into $g$, we obtain the following expression for the interaction Hamiltonian :

$$
\mathcal{H}_{1}=g_{1} \int_{t_{0}}^{\infty} \mathrm{d} t \int_{t_{0}}^{t} \mathrm{~d} t_{1} \int \mathrm{d} \mathbf{r} \hat{\psi}(\mathbf{r}, t) \psi(\mathbf{r}, t) \hat{\psi}\left(\mathbf{r}, t_{1}\right) \psi\left(\mathbf{r}, t_{1}\right) .
$$


Let us now compute in perturbation theory the Laplace (time) and Fourier (space) transform of $G$. We then obtain graphs which are in one-to-one correspondence with those appearing in the computation of the equilibrium conformation of a self-repelling chain [10]. One only has to redefine the coupling constant by a numerical factor and to interpret the Laplace parameter $\mu$ as the monomer chemical potential of the self-repelling chain.

This result is not surprising. We are in fact summing over all paths leading from $r_{0}$ to $r$ in the time $t-t_{0}$, but assigning to paths which have $n$ intersections a Boltzmann factor of the form $\exp (-g n)$. We have therefore obtained a kinetic description of the self-avoiding walk (SAW; also called the self-repelling chain). The static information contained in the theory of the SAW [10] can be now interpreted from a kinetic point of view. The shift $\mu_{\mathrm{c}}$ of the critical value of $\mu$ corresponds for instance to the exponential decay $\exp \left(-\mu_{\mathrm{c}} t\right)$ of the probability of a SAW of length $t$ to survive.

Let us now generalize the model, introducing a tendency of the walk to avoid crowded areas. This corresponds to adding to the interaction Hamiltonian (Eq. (7)) a term corresponding to the interaction term of the TSAW [6].

We have :

$$
\mathscr{H}_{2}=-g_{2} \int_{t_{0}}^{\infty} \mathrm{d} t \int_{t_{0}}^{t} \mathrm{~d} t_{1} \int \mathrm{dr}\left(\nabla\left(\hat{\psi}\left(\mathbf{r}, t_{1}\right) \psi\left(\mathbf{r}, t_{1}\right)\right) \cdot \nabla \hat{\psi}(\mathbf{r}, t)\right) \psi(\mathbf{r}, t) .
$$

Simple power counting shows nevertheless that this interaction is irrelevant (at least in $D=4-\varepsilon$ ) when the interaction (7) is present. Although it surely contributes to make walks live longer it cannot modify their asymptotic behaviour.

\section{Kinetic growth walks.}

It is not easy to justify the form of an interaction Hamiltonian describing the property of the walks to terminate whenever they end up in a lattice site with no unvisited neighbours. Majid et al. [2] suggest that the trap density is in this case proportional to some power of the density of visited points $\rho$. They specify the law $\phi \propto \rho^{2}$. This would lead to the interaction Hamiltonian

$$
\mathscr{H}_{3}=g_{3} \int_{t_{0}}^{\infty} \mathrm{d} t \int_{t_{0}}^{t} \mathrm{~d} t_{1} \int_{t_{0}}^{t} \mathrm{~d} t_{2} \int \mathrm{dr}\left(\hat{\psi}\left(\mathbf{r}, t_{1}\right) \psi\left(\mathbf{r}, t_{1}\right)\right)\left(\hat{\psi}\left(\mathbf{r}, t_{2}\right) \psi\left(\mathbf{r}, t_{2}\right)\right)(\hat{\psi}(\mathbf{r}, t) \psi(\mathbf{r}, t)) .
$$

Majid et al. [2] then use a Flory argument to investigate the asymptotic behaviour of such a model, and obtain the same results as in the Flory theory of a single polymer chain at the $\theta$ point [10]. They then suggest that this result should be valid even beyond the Flory approximation.

Diagrams obtained from this interaction do indeed correspond to those of the equilibrium properties of polymers at the point where direct monomer-monomer interactions vanish. This is not the same as the $\theta$-point, which is the point where the second virial coefficient of an isolated polymer chain vanishes. In the language of the $n=0$ vector model of isolated polymer chains [10] the first situation corresponds to the vanishing of the bare four-point interaction, whereas the second corresponds to the vanishing of the true four-point vertex.

If we accept the guess that the density of traps is proportional to the square of $\rho$, we are thus led to the conclusion that the kinetic growth walk belongs to the same universality class as the SAW, at least in $D=4-\varepsilon$, and taking into account that there may be some exceptional value of the coupling constant for which the walk falls in a more restricted universality class.

A way of making this result more palatable is to consider that since the tendency of the walk to avoid places already visited is irrelevant, this tendency may be disposed of altogether. The 
question then boils down to understanding the distribution of traps generated by an ordinary random walk in its development. Most of them,will appear when portions of the walk corresponding to widely separated time regions come together in space (this corresponds to $\phi \propto \rho^{2}$ ). Nothing prevents however traps to be formed by crooked configurations of the path over a short time interval. No matter how rare these traps are, they contribute to an interaction of the form of equation (7) which modifies the asymptotic behaviour even within the Flory argument.

\section{Discussion.}

We obtain the conclusion that the asymptotic behaviour of the KGW is the same as that of the kinetic version of the SAW. The simulation data for walks in three dimensions [2] are not in flat contradiction with this conclusion if one argues that the trap density in three dimensions is by any means very small and that one is only witnessing the very first departure from an ordinary random walk behaviour (even if the TSAW effect is irrelevant, it contributes to screen the interaction). What is more worrying is the universal behaviour in two dimensions, at least for the path lengths shown. It is possible that in this case the conclusions above do not apply much in the same way as the one-dimensional TSAW with infinite interaction behaves differently from the TSAW with a finite interaction. Or may be one is looking at a quasi-scaling behaviour due to an intermediate fixed point. Checks of the universality of the behaviour with respect to $g$ may help to understand the question.

\section{Acknowledgments.}

It is a pleasure to thank L. Pietronero and G. Parisi for stimulating conversations and A. Coniglio for a useful discussion.

\section{References}

[1] Amit, D. J., Parisi, G., Peliti, L., Phys. Rev. B 27 (1983) 1635.

[2] Majid, I., Jan, N., Coniglio, A., Stanley, H. E., Phys. Rev. Lett. 52 (1984) 1257.

[3] LyKLema, J. W., KREMER, K., to be published.

[4] Kremer, K., LyKlema, J. W., to be published.

[5] Obukhov, S. P., Peliti, L., J. Phys. A 16 (1983) L-147.

[6] Peliti, L., in : C. Itzykson, Y. Pomeau, N. Sourlas (eds.), Phys. Rep. 103 (1984) 225.

[7] LuCK, J. M., Nucl. Phys. B 225 (1983) 169.

[8] Bugadaev, S. A., Obukhov, S. P., to be published.

[9] Martin, P. C., Siggia, E. D., Rose, H. A., Phys. Rev. A 7 (1973) 423.

[10] De Gennes, P. G., Scaling Concepts in Polymer Physics (Ithaca, N.Y. : Cornell University Press) 1979. 